\title{
Nonleptonic Kaon Decays from Lattice QCD
}

\author{
Norman Christ ${ }^{* \dagger}$ \\ Columbia University, USA \\ E-mail: nhcephys.columbia.edu
}

Improved methods and increased computer capabilities now allow direct calculation of the decay of the kaon into two pions to be carried out using lattice QCD. Recent results for the complex, $I=2, K \rightarrow \pi \pi$ amplitude $A_{2}$ with physical kinematics will be presented. Initial studies of the more difficult $I=0$ amplitude $A_{0}$ will also be described. These studies give insight into the $\Delta I=1 / 2$ rule and, when combined with new methods that will also be discussed, lay the foundation for an accurate calculation of the direct $\mathrm{CP}$ violating parameter $\varepsilon^{\prime}$.

2013 Kaon Physics International Conference

Apr 29-30 and May 1st

University of Michigan, Ann Arbor, Michigan - USA

\footnotetext{
* Speaker.

${ }^{\dagger}$ This work was supported in part by US DOE grant DE-FG02-92ER40699.
} 


\section{Introduction}

Substantial advances in algorithms and high-performance computers now allow lattice QCD calculations that were not possible even a year or two ago. An important threshold that has been crossed recently is the ability to perform calculations in which the light quarks and therefore the pions have their physical masses. This step has two important consequences. Lattice calculations of familiar quantities such as particle masses, pseudoscalar decay constants and bag parameters can now be computed directly without the need to make uncertain assumptions about the accuracy of chiral perturbation theory that were previously necessary: A significant source of systematic error present in lattice calculation has been simply removed. A second important consequence of the ability to work at physical quark mass is that we can attempt to compute more complex quantities now made simpler because we need not deal with the effects of unphysical masses. The calculation

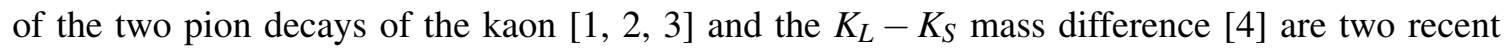
efforts undertaken to exploit this important advance.

Here we will discuss the status and prospects for computing the $I=0$ and $2, K \rightarrow \pi \pi$ decay amplitudes $A_{0}$ and $A_{2}$ from first-principles using lattice techniques. We begin by describing the methods that allow these physical decay amplitudes to be computed using lattice methods. This discussion is then specialized to the easier case of complex amplitude $A_{2}$ where results for the real and imaginary parts using physical kinematics are now available. Next we turn to the more difficult case of $A_{0}$ and describe the state of recent numerical experiments, an array of improved techniques that offer substantial reductions in computational costs and the calculation using physical kinematics that has now begun. Finally the recent RBC/UKQCD lattice QCD insights into the $\Delta I=1 / 2$ rule will be discussed.

\section{2. $K \rightarrow \pi \pi$ decays on a four-dimensional Euclidean lattice}

More than ten years ago, Lellouch and Luscher [[] recognized that the Maiani-Testa "no-go" theorem [ 6 , impeding the calculation of two-body decays using lattice QCD, could be circumvented by exploiting the finite volume in which lattice calculations are performed. In a standard lattice calculation an operator is introduced with the quantum numbers needed to create a particular physical state and then a sufficient time extent is introduced so that the single lowest energy state with those quantum numbers results. For the case of two pions, this lowest energy state will be two pions at rest (for $I=2$ ) or, even worse, the vacuum $(I=0)$. Lellouch and Luscher pointed out in finite volume there will be a series of discrete QCD energy eigenstates corresponding approximately to states where the components of the pion momenta are integer multiples of $2 \pi / L$. Thus, by tuning the spatial size of the box in which the calculation is performed, we can arrange that one of these excited two-pion states has an energy which matches that of the kaon. The matrix element of the effective $\Delta S=1$ weak Hamiltonian between the kaon and this excited state then agrees with the on-shell, infinite volume physical matrix element up to a finite-volume correction, which Lellouch and Luscher show can be determined by lattice methods. This is illustrated in Fig. W, which shows schematically a pion wave function with momentum $p \approx 2 \pi / L$. This well-controlled theoretical analysis permits the direct lattice calculation of $A_{0}$ and $A_{2}$ to sub-percent precision, although electromagnetic effects will likely have to be included to achieve this level of accuracy. 


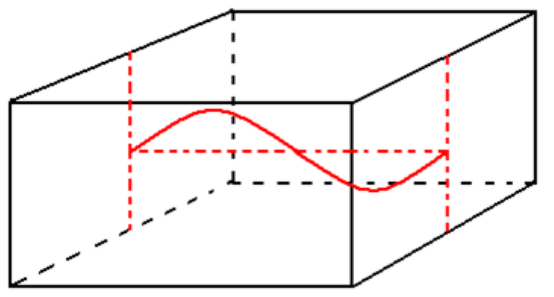

Figure 1: An illustration of the LellouchLuscher, finite-volume approach to $K \rightarrow \pi \pi$ decay. The size of the box is adjusted so that the energy of a finite-volume $\pi-\pi$ state coincides with the kaon mass.

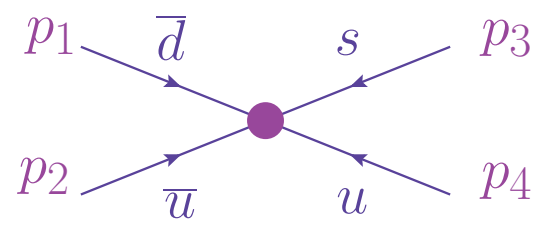

Figure 2: A Feynman diagram representing the four-point functions $\Gamma_{i}\left(p_{1}, p_{2}, p_{3}, p_{4}\right)$ appearing in Eq. (2.2) upon which the RI renormalization conditions are imposed.

While theoretically sound this method is computationally challenging because it requires distinguishing a term which falls exponentially with increasing time relative to the unphysical state in which the pions have near-zero momentum, or for the more difficult $I=0$ state, distinguishing a term falling exponentially with increasing time relative to both the zero-momentum $\pi-\pi$ and the vacuum states! This issue is represented by the equation for the dependence of the correlation function $G(t)$ on the time separation $t$ between the weak Hamiltonian which creates the two-pion final state and the operator which destroys these two pions:

$$
G(t)=c_{0}+c_{1} e^{-E_{1} t}+c_{2} e^{-E_{2} t} .
$$

Here we assume $E_{2}>E_{1}$. For the $I=0$ case all three terms are present. The constant term $c_{0}$ is the vacuum contribution while $c_{1}$ describes the two-pion state at rest. For a properly chosen volume the third term $c_{2}$ contains the decay amplitude of interest. However, at large times where we may hope to precisely distinguish these three terms the $c_{2}$ piece will be very small and difficult to accurately determine. The $I=2$ case is somewhat better because the vacuum term, $c_{0}$ is absent.

This difficulty can be substantially reduced by imposing anti-periodic boundary conditions on the pions. For the $I=0$ final state this can be done by imposing G-parity boundary conditions on the quarks [ [ $]$ ] while for the $I=2, \pi-\pi$ state we need only impose anti-periodic boundary conditions on the $d$ quark [ [ $]$ ]. The spatial size of the lattice volume and the number of spatial directions in which these boundary conditions are imposed can be chosen so that the finite-volume two-pion state with the lowest energy is degenerate with the initial kaon. For the case of $I=2$ this implies that in the large time limit the leading term is the one describing the physical $K \rightarrow \pi \pi$ decay. For the $I=0$ case the leading large-time behavior corresponds to the unphysical $K \rightarrow|\mathrm{vac}\rangle$ transition. However, the next-leading term will describe the physical $I=0, K \rightarrow \pi \pi$ decay.

A second challenge that must be addressed is the determination of the lattice expression for the electro-weak operator which causes the decay. While the general methods needed to accomplish this task have been in use for some time [ $[$ ], there have been important refinement which substantially increase the accuracy of the method. The starting point in creating an appropriate lattice weak decay Hamiltonian is the procedure, already highly developed in the 1970's, of representing the fundamental $W$ exchange process by an effective Hamiltonian that when used in low-energy matrix elements, such as those that must be computed in a lattice calculation, reproduces the results of the original theory. The determination of this effective weak operator in continuum field 
theory is described in the review article of Buchalla, et al. [ए]] and results in a specific combination of seven independent, dimension-six, four-quark operators normalized in the $\overline{\mathrm{MS}}$ scheme.

These operators, defined in the continuum, are converted into lattice operators in two steps. First a continuum QCD perturbation theory calculation is performed to convert from the $\overline{\mathrm{MS}}$ renormalization scheme which requires dimensional regularization to the Rome/Southampton, regularization independent (RI) scheme which can be imposed in both the continuum and lattice theories. Here we define the normalization and the mixing of these seven operators by imposing conditions on off-shell, Landau gauge-fixed, Green's functions in which these operators appear. Schematically we evaluate seven momentum-space Green's functions corresponding to these seven independent operators as shown in Fig. \, determining the amplitudes:

$$
\left(\Gamma_{i}\left(p_{1}, p_{2}, p_{3}, p_{4}\right)\right)_{a b c d}^{\alpha \beta \gamma \delta}=\prod_{j=1}^{4}\left(\int d^{4} x_{j} e^{i p_{j} \cdot x_{j}}\right)\left\langle\bar{q}_{a}^{\alpha}\left(x_{1}\right) \bar{q}_{b}^{\beta}\left(x_{2}\right) Q_{i} q_{c}^{\gamma}\left(x_{3}\right) q_{d}^{\delta}\left(x_{4}\right)\right\rangle,
$$

where $\alpha, \beta, \gamma$ and $\delta$ are spin indices, $a, b, c$ and $d$ are color indices and $1 \leq i \leq 7$. The RI operators are then defined by imposing the conditions

$$
\operatorname{Tr}\left\{P_{k} \Gamma_{i}\left(p_{1}, p_{2}, p_{3}, p_{4}\right)\right\}=F_{k i} .
$$

Here $\operatorname{Tr}$ indicates a sum over the flavor and spin indices, the $P_{k}, 1 \leq k \leq 7$ are seven projection operators and $F_{k i}$ the results obtained if these conditions are applied in the free-field case. The momenta $p_{1}, p_{2}, p_{3}$ and $p_{4}$ are fixed at non-exceptional values to insure that the amplitudes $\Gamma_{i}$ are infrared safe and can be reliably computed in perturbation theory if the scale of these momenta is sufficiently large [], [2]. We can the apply the conditions in Eq. ([2.3]) to normalize our lattice operators without the use of perturbation theory.

Two recent developments bring this process under more precise control. First, using these RI conditions we can define physically equivalent operators on multiple gauge ensembles with lattice spacings varying between the larger values needed when simulating physical-mass pions and much smaller values needed to reduce, to whatever extent desired, the perturbation series truncation errors that arise when the continuum effective Hamiltonian is determined and the $\overline{\mathrm{MS}}$ and RI scheme related [[13]. This technique can essentially eliminate the errors associated with the use of perturbation theory. Second, we can use twisted boundary conditions for the fermion fields, allowing momenta which are non-integer multiples of $2 \pi / L$ when imposing the RI conditions in Eq. (‥3) [[1]3]. This permits the lattice four-momenta to be continuously varied while the orientations of these four-momenta relative to the lattice directions are kept fixed. This permits an accurate continuum limit to be taken, avoiding variations in the $O\left(a^{2}\right)$ terms that would result if the orientations of the momenta are changed and when the lattice spacing is changed. Using these methods and presuming adequate computer resources, the accuracy of such a lattice QCD calculation of $K \rightarrow \pi \pi$ decay is limited by the most important phenomena that have been omitted: electromagnetic and other isospin breaking effects which might be expected to enter at the percent level for $A_{0}$ and at a possible larger relative value for the accidentally suppressed amplitude $A_{2}$.

Central to the $K \rightarrow \pi \pi$ calculations described below is the use of a chiral fermion formulation, in our case domain wall fermions [14, [5]. While all fermion formulations are expected to show chiral behavior at long distances, chiral fermions have a short-distance behavior which also respects 
chiral symmetry. This reduces the number of operators that must be introduced in the effective electro-weak theory and greatly simplifies the mixing of those operators, a simplification that is critical to the application of the renormalization methods described above. In addition, this physical character of the domain wall formulation at short distances leads to relatively small discretization errors, reducing the errors associated with evaluating the continuum limit [ए6]].

\section{3. $K \rightarrow \pi \pi$ decay, $\Delta I=3 / 2$}

The first realistic, $a b$ initio calculation of the amplitude $A_{2}$ was completed in 2011 [ [2], [3]. The $I=2$ final state does not receive a vacuum contribution and anti-periodic boundary conditions can be easily imposed on the two final-state pions by using isospin symmetry to relate the physical decay to one in which the two final-state pions are both $\pi^{+}$. These $\pi^{+}$mesons can be made to obey anti-periodic boundary conditions by imposing anti-periodic boundary conditions on the $\bar{d}$ quarks and periodic boundary condition on the $u$ quarks. This calculation used a kaon and pions with essentially physical masses, 505.5(3.4) MeV and 142.11(94), respectively, while the energy of the two-pion final state had the close to energy conserving value of $485.5(4.2) \mathrm{MeV}$. The result for the complex amplitude $A_{2}$ was:

$$
\begin{aligned}
& \operatorname{Re}\left(A_{2}\right)=1.381(46)_{\text {stat }}(258)_{\text {syst }} \times 10^{-8} \mathrm{GeV} \\
& \operatorname{Im}\left(A_{2}\right)=6.54(46)_{\text {stat }}(120)_{\text {syst }} \times 10^{-13} \mathrm{GeV}
\end{aligned}
$$

where the large systematic error is dominated by our estimate of the discretization errors arising from the single and relatively small inverse lattice spacing of $1 / a=1.371(8) \mathrm{GeV}$ used.

This calculation was carried out on a specially designed $32^{3} \times 64$ ensemble of gauge fields whose large lattice spacing was chosen to give a sufficiently large volume that pions of physical mass could be studied. However, such a coarse lattice spacing gives rise to large residual chiral symmetry breaking for the domain wall fermion formulation and an extra dislocation suppressing determinant ratio (DSDR), developed for the study of QCD thermodynamics, was added to the gauge action to allow physical quark masses to be studied [ㄱ]].

Fortunately, enormous progress in numerical algorithms and computer hardware has taken place since this first $K \rightarrow \pi \pi$ calculation was begun in 2010. What was a large pioneering effort has now been made routine by using the all mode averaging technique [ए8] and the large IBM Blue Gene/Q Mira computer at Argonne and the smaller Blue Gene/Q machines at the RIKEN BNL Research Center, the Brookhaven National Laboratory and the University of Edinburgh. The original $32^{3} \times 64$ volume has been increased to $48^{3} \times 96$ and $64^{3} \times 128$ and the relatively small inverse lattice spacing of $1.37 \mathrm{GeV}$ has been replaced by the two larger values of 1.73 and 2.28 $\mathrm{GeV}$, allowing a continuum limit to be evaluated. While still preliminary, first results were reported (after this talk was given) at Lattice 2013 by Tadesuz Janowski:

$$
\begin{aligned}
& \operatorname{Re}\left(A_{2}\right)=1.345(84)_{\text {stat }} \times 10^{-8} \mathrm{GeV} \\
& \operatorname{Im}\left(A_{2}\right)=6.32(28)_{\text {stat }} \times 10^{-13} \mathrm{GeV} .
\end{aligned}
$$

These results now come from an extrapolation to the continuum limit and are expected to have systematic errors smaller than those in Eq. (B. (1) by factor of two. The agreement with our earlier 
results from an independent ensemble and the agreement of $\operatorname{Re}\left(A_{2}\right)$ with the experimental values $\operatorname{Re}\left(A_{2}\right)=1.479(4)$ and $1.573 \times 10^{-8}$ (obtained from $K^{+}$and $K_{S}$ decays respectively) is very good.

The value for $\operatorname{Im}\left(A_{2}\right)$ was previously unknown. Assuming the standard model, we can combine the results in Eq. (B.] $)$ with the experimental result for $\operatorname{Re}\left(A_{0}\right)$ and $\operatorname{Re}\left(\varepsilon^{\prime} / \varepsilon\right)$ to determine the two $\mathrm{CP}$ violating ratios whose difference determines the direct $\mathrm{CP}$ violation parameter $\varepsilon^{\prime}$ :

$$
\begin{aligned}
& \frac{\operatorname{Im}\left(A_{2}\right)}{\operatorname{Re}\left(A_{2}\right)}=-4.76(37)_{\text {stat }}(81)_{\text {syst }} \times 10^{-5} \\
& \frac{\operatorname{Im}\left(A_{0}\right)}{\operatorname{Re}\left(A_{0}\right)}=-16.3(1.9)_{\text {stat }}(20)_{\text {syst }} \times 10^{-5},
\end{aligned}
$$

showing that $\varepsilon^{\prime}$ is dominated by the contribution of $\operatorname{Im}\left(A_{0}\right)$, without large cancellation between the $I=0$ and $I=2$ contributions. This calculation is now part of the standard "measurement" package of RBC/UKQCD and will be refined future calculations in much the way as the calculation of the pseudoscalar decay constants $f_{\pi}$ and $f_{K}$. We now turn to the much more difficult lattice calculation $A_{0}$ and the challenge of a complete lattice calculation of the standard model value for $\varepsilon^{\prime}$.

\section{4. $K \rightarrow \pi \pi$ decay, $\Delta I=1 / 2$}

Both $A_{0}$ and $A_{2}$ are needed for a complete description of $K \rightarrow \pi \pi$ decay, making the calculation of the remaining, $I=0$ amplitude, $A_{0}$, a high priority. However, this is the more difficult of the two to calculate since the $I=0 \pi-\pi$ state has vacuum quantum numbers, implying that some of the needed amplitudes will have only gluons connected the initial and final quarks. For such amplitudes the exponential decay with increasing Euclidean time separation that identifies the energies of the states being studied is not realized by the decreasing size of the quark propagators joining the initial and final states and the intermediate weak decay Hamiltonian. Instead, this exponential decrease comes from the increasingly complete cancelation of individual path integral contributions which themselves do not decrease. As a result such "quark-line-disconnected" amplitudes are plagued with large statistical noise and very large statistical samples are needed.

We are pursuing two strategies to overcome this challenge. First, we must collect large statistics. The first calculation to detect a non-zero result for $\operatorname{Re}\left(A_{0}\right)$ using unphysical threshold kinematics [四] studied 800 configurations and performed 32 independent measurements on each. Second, we can construct an operator which destroys the $\pi-\pi$ final state but which couples weakly to the vacuum. (The long-time amplitude which must be canceled by the Monte Carlo average is the amplitude describing the coupling to the vacuum intermediate state.) At present we have two complementary methods to do this. In the first we locate the quark fields associated with each of the two pions on separate time slices, where a separation of four appears to work best. This allowed a calculation of the more difficult $\operatorname{Im}\left(A_{0}\right)$, again at threshold with unphysical masses, from 138 configurations [미, [20]. This "split-source" method removes the terms where a quark-anti-quark pair, one from each pion, can immediately annihilate - behavior enhancing the $\langle\pi \pi|$ vac $\rangle$ overlap.

The second refinement of the $\pi-\pi$ operator uses all-to-all methods [2]] to introduce localized quark-anti-quark wave functions for each pion, enhancing the coupling to the target two-pion state while removing large parts of the $\pi-\pi$ operator which couple only to the vacuum. Results presented at Lattice 2013 by Daiqian Zhang, show an improvement equivalent to a 4-5 times increase in statistics. 
A second challenge offered by the $I=0$ state is the introduction of anti-periodic boundary conditions for the pion. Here we use an idea of Changhoan Kim [ [ 22] and impose G-parity boundary conditions. These are unusual boundary conditions which require the introduction of two explicit flavors of light quarks and the imposition of boundary conditions under which a $u$ quark becomes a $\bar{d}$ and a $d$ becomes a $-\bar{u}$ upon passing through a spatial boundary in one or more directions. These boundary conditions preserve isospin (so the identification of $\mathrm{I}=0$ and $\mathrm{I}=2$ states still makes sense) and can be used to insure that the lowest, finite volume $\pi-\pi$ energy equals $M_{K}$. The charge-conjugation in this identification requires that the gauge field also obeys chargeconjugation boundary conditions, so new, special gauge-configurations must be generated. Thus, both the measurement code and the code generating the gauge configurations must be modified to incorporate the two distinct fermion flavors and these charge-conjugation boundary conditions. This is made more difficult because this must remain highly efficient, high-performance code. This has been accomplished. Again at Lattice 2013, Christopher Kelly announced the beginning of fullscale simulations with G-parity boundary conditions which will allow the first calculation of the $I=0$ amplitude $A_{0}$ with physics kinematics on an ensemble of gauge configurations very similar to those used for the first, physical calculation of $A_{2}$. We hope to have preliminary results from this exploratory within a year and more definitive results in possibly two years. While the expected accuracy cannot be confidently estimated until we have a better understanding of the required statistics, our goal is a $20 \%$ result, similar to our initial result for $A_{2}$.

\section{The $\Delta I=1 / 2$ rule}

As a final topic, we will discuss the understanding of the $\Delta I=1 / 2$ rule that has recently emerged from the physical results for $A_{2}$ and the first calculations of $A_{0}$ with unphysical kinematics [23]. (The reader is referred to this paper for references to earlier work on this topic.) Recall that the $\Delta I=1 / 2$ rule refers to the large ratio found for the $I=0$ and $I=2$ amplitudes: $\operatorname{Re}\left(A_{0}\right) / \operatorname{Re}\left(A_{2}\right)=22.4$. For our two sets of unphysical, threshold kinematics [U, प9, [20] we can compute this ratio and obtain:

$$
\begin{array}{lll}
\frac{\operatorname{Re}\left(A_{0}\right)}{\operatorname{Re}\left(A_{2}\right)}=9.1(21) & M_{\pi}=422 \mathrm{MeV} & M_{K}=877 \mathrm{MeV} \\
\frac{\operatorname{Re}\left(A_{0}\right)}{\operatorname{Re}\left(A_{2}\right)}=12.0(17) & M_{\pi}=329 \mathrm{MeV} & M_{K}=622 \mathrm{MeV}
\end{array}
$$

While not equal to the experimental value of 22.5 , these numbers are substantially larger than the ratio of approximately 2 given by the Wilson coefficients alone. In addition, as we decrease the pion mass towards its physical value, the ratio is seen to increase.

Further evidence that our current calculations may have captured the essence of the $\Delta I=1 / 2$ rule is shown by Fig. [3]. There we plot both the lattice results for $\operatorname{Re}\left(A_{2}\right)$ computed with essentially physical kinematics as well as results for $\operatorname{Re}\left(A_{2}\right)$ and $\operatorname{Re}\left(A_{0}\right)$ computed for a threshold $\pi-\pi$ final state and a pion with mass 422 and $329 \mathrm{MeV}$, results presented in Refs. [U] and [ए耳, [20] respectively. We see that $\operatorname{Re}\left(A_{2}\right)$ decreases dramatically with increasingly physical kinematics and agrees well with the physical value at the physical point. For $\operatorname{Re}\left(A_{0}\right)$ no physical calculation has yet been performed but for the two unphysical cases studied the results are similar and close to the physical result. 


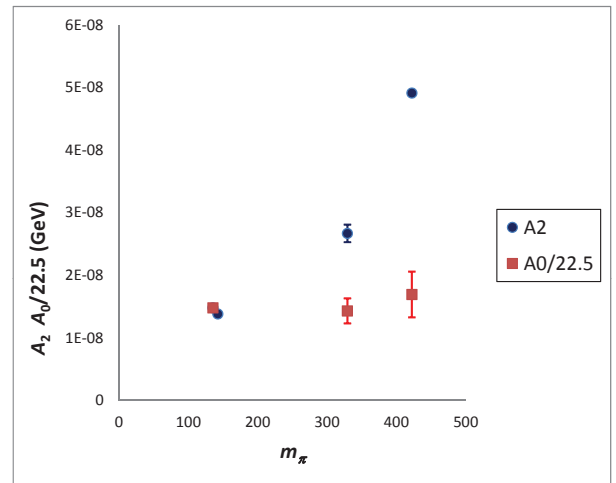

Figure 3: Our results for the $\operatorname{Re}\left(A_{2}\right)$ computed for both threshold decays with $m_{\pi}=$ 329 and $422 \mathrm{MeV}$ and for physical kinematics together with those for $\operatorname{Re}\left(A_{0}\right)$ where only the two points with threshold kinematics are available.
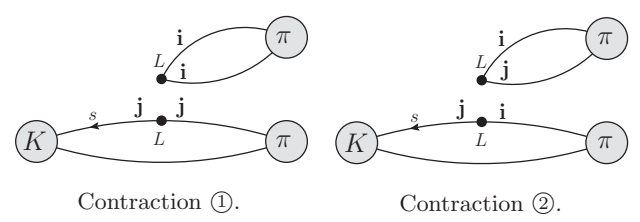

Figure 4: The two types of contractions which give the largest contributions to the real parts of $A_{0}$ and $A_{2}$. For the case of $A_{0}$ they add while for $A_{2}$ they cancel.

Assuming that a calculation $\operatorname{Re}\left(A_{0}\right)$ with physical kinematics is similar to those that we have performed, we can examine our calculations of $\operatorname{Re}\left(A_{2}\right)$ and $\operatorname{Re}\left(A_{0}\right)$ to identify the mechanism responsible for the $\Delta I=1 / 2$ rule. The current-current operator $Q_{2}$ gives the dominant contributions to $\operatorname{Re}\left(A_{2}\right)$ and $\operatorname{Re}\left(A_{0}\right)$ and the corresponding diagrams, labeled as (1) and (2), are shown in Fig. 田. These combine to give the $Q_{2}$ contribution to $A_{2}$ and $A_{0}$ as

$$
\begin{aligned}
& A_{2}=\sqrt{\frac{2}{3}}((1)+(2)) \\
& A_{0}=\sqrt{\frac{1}{3}}(2 \cdot(1)-(2) .
\end{aligned}
$$

For the case of free fields or if we assume that current-current matrix element between a kaon and two pions factorizes into a product of a $K-\pi$ matrix element of one current times the $\pi-|\mathrm{vac}\rangle$ matrix element of the other, then (2) $\simeq \frac{1}{3}$ (1) causing these two contribution to add for $A_{2}$ and subtract for $A_{0}$. In fact this factorization hypothesis does not describe our numerical results which give (2) $\simeq-0.75$ (1). (This is also poorly described by a large $N$ approximation in which the color mixed amplitude (2) would be neglected.)

Thus, our calculation suggests that the $\Delta I=1 / 2$ rule arises from the cancellation of two similar term which contribute to both $A_{2}$ and $A_{0}$. These largely cancel when combined to give $A_{2}$ resulting in a combination which is sensitive to the quark masses and causes $A_{2}$ to decrease by more than a factor of 3 between our $m_{\pi}=422$ and $142 \mathrm{MeV}$ results. We find this cancellation to be the largest for the case of physical kinematics. In contrast, these amplitudes add to give $A_{0}$, resulting in an amplitude that appears less sensitive to the quark masses and is not far from its physical value, even for our unphysical kinematics. The final piece of this "explanation" of the $\Delta I=1 / 2$ rule will be the actual calculation of $A_{0}$ for physical kinematics, a project now underway.

While it is best left to the reader to judge if this can be properly called an explanation of the $\Delta I=1 / 2$ rule, the mechanism uncovered does have consequences. Since this large experimental 
ratio of 22.5 appears to follow from the standard model, no new physics needs to be introduced to explain it. Since $A_{2}$ is made small by an apparently accidental cancellation, other processes contributing to $A_{2}$ might be expected not to show a similar suppression. This is certainly the case for the relatively large CP-violating phase of $A_{2}$ arising from the electroweak penguin contribution, which is not similarly suppressed. Likewise, we might expect that the electromagnetic contributions to $\operatorname{Re}\left(A_{2}\right)$ will be relatively large since the normal $\alpha_{\mathrm{EM}}$ terms will likely be free of this $1 / 22.5$ suppression factor.

\section{Outlook}

The lattice QCD treatment of the weak interactions of the quarks has made enormous progress since the first calculations were attempted in the 1980's. With the refinement of methods to impose continuum normalization on lattice operators, the algorithmic and computational capability to work with physical values for the light quark masses and the techniques needed to accurately describe interacting, two-pion states above threshold, it is now possible to study the weak interactions of $K$ mesons with few-percent-level precision. Such results are available now for the easier $\Delta I=3 / 2$ amplitude $A_{2}$ now and similar results for the more difficult amplitude $\Delta I=1 / 2 A_{0}$ in 2-3 years. On a 2-5 year time scale we may also anticipate the accurate inclusion of charm quark loops in such kaon amplitudes. This will allow similarly accurate treatment of the $K_{L}-K_{S}$ mass difference [四] and the contribution of charm quarks to rare kaon decays. Such a non-perturbative treatment of charm may also be required for the accurate calculation of the imaginary part of $A_{0}$. At present this is computed using QCD perturbation theory to "integrate out" the charm quark, an approach with uncertain accuracy. Errors arising from the truncation of the perturbative series or from the neglect of $\left(\Lambda_{\mathrm{QCD}} / m_{c}\right)^{2}$ corrections may require the non-perturbative inclusion of charm to reach percent-level accuracy for a quantity such as $\varepsilon^{\prime}$.

The author thanks his RBC/UKQCD collaborators with whom this work was done. Critical to this calculation were the Blue Gene/P and /Q computers at the Argonne Leadership Computing Facility (DOE contract DE-AC02-06CH11357). Also important were the DOE USQCD and RIKEN BNL Research Center QCDOC and Blue Gene/Q computers at the Brookhaven National Lab., the Blue Gene/Q computer at the DiRAC facility and the University of Southampton's Iridis cluster.

\section{References}

[1] T. Blum, P. Boyle, N. Christ, N. Garron, E. Goode et al., $K$ to $\pi \pi$ Decay amplitudes from Lattice QCD, Phys.Rev. D84 (2011) 114503 [arXiv:1106.2714 hep-lat].

[2] T. Blum, P. Boyle, N. Christ, N. Garron, E. Goode et al., The $K \rightarrow(\pi \pi)_{I=2}$ Decay Amplitude from Lattice QCD, Phys.Rev.Lett. 108 (2012) 141601 [arXiv:1111.1699 [hep-lat]].

[3] T. Blum, P. Boyle, N. Christ, N. Garron, E. Goode et al., Lattice determination of the $K \rightarrow(\pi \pi)_{I=2}$ Decay Amplitude A2, Phys.Rev. D86 (2012) 074513 [arXiv:1206.5142 [hep-lat]].

[4] N. Christ, T. Izubuchi, C. Sachrajda, A. Soni and J. Yu, Long distance contribution to the $K_{L}-K_{S}$ mass difference, Phys. Rev. D88 (2012) 014508 [arXiv:1212.5931 hep-lat]].

[5] L. Lellouch and M. Luscher, Weak transition matrix elements from finite-volume correlation functions, Commun. Math. Phys. 219 (2001) 31-44 [hep-1at/0003023]. 
[6] L. Maiani and M. Testa, Final state interactions from euclidean correlation functions, Phys. Lett. B245 (1990) 585-590.

[7] C.-h. Kim and N. H. Christ, $K \rightarrow>$ pi pi decay amplitudes from the lattice, Nucl. Phys. Proc. Suppl. 119 (2003) 365-367 [hep-lat/0210003].

[8] C. Kim, I = 2 pi pi scattering using G-parity boundary condition, Nucl. Phys. Proc. Suppl. 129 (2004) 197-199 [arXiv:hep-1at/0311003].

[9] RBC Collaboration, T. Blum et al., Kaon matrix elements and CP-violation from quenched lattice QCD I: The 3-flavor case, Phys. Rev. D68 (2003) 114506 [hep-1at/0110075].

[10] G. Buchalla, A. J. Buras and M. E. Lautenbacher, Weak decays beyond leading logarithms, Rev. Mod. Phys. 68 (1996) 1125-1144 [hep-ph/9512380].

[11] Y. Aoki, P. Boyle, N. Christ, C. Dawson, M. Donnellan et al., Non-perturbative renormalization of quark bilinear operators and $B_{K}$ using domain wall fermions, Phys.Rev. D78 (2008) 054510 [arXiv:0712.1061 [hep-lat]].

[12] C. Sturm et al., Renormalization of quark bilinear operators in a momentum- subtraction scheme with a nonexceptional subtraction point, Phys. Rev. D80 (2009) 014501 [arXiv:0901.2599 एhep-ph].

[13] RBC, UKQCD Collaboration, R. Arthur and P. Boyle, Step Scaling with off-shell renormalisation, Phys.Rev. D83 (2011) 114511 [arXiv:1006.0422 [hep-lat]].

[14] D. B. Kaplan, A method for simulating chiral fermions on the lattice, Phys. Lett. $\mathbf{B 2 8 8}$ (1992) 342-347 [hep-1at/9206013].

[15] Y. Shamir, Chiral fermions from lattice boundaries, Nucl. Phys. B406 (1993) 90-106 [hep-1at/9303005].

[16] RBC, UKQCD Collaboration, Y. Aoki et al., Continuum Limit Physics from 2+1 Flavor Domain Wall QCD, Phys.Rev. D83 (2011) 074508 [arXiv:1011.0892 [hep-lat]].

[17] D. Renfrew, T. Blum, N. Christ, R. Mawhinney and P. Vranas, Controlling Residual Chiral Symmetry Breaking in Domain Wall Fermion Simulations, arXiv:0902.2587 hep-lat].

[18] T. Blum, T. Izubuchi and E. Shintani, A new class of variance reduction techniques using lattice symmetries, arXiv:1208.4349 [hep-lat].

[19] Q. Liu, Practical methods for a direct calculation of $\Delta I=1 / 2 K$ to $\pi \pi$ Decay, PoS LATTICE2011 (2011) 287 [arXiv:1110.2143 hep-lat]].

[20] Q. Liu, Kaon to two pions decays from lattice $Q C D: \Delta I=1 / 2$ rule and CP violation, . ISBN: 9781267290649.

[21] J. Foley, K. Jimmy Juge, A. O'Cais, M. Peardon, S. M. Ryan et al., Practical all-to-all propagators for lattice QCD, Comput.Phys.Commun. 172 (2005) 145-162 [arXiv:hep-lat/0505023 [hep-Iat].

[22] C. Kim and N. H. Christ, G parity boundary conditions and Delta $I=1 / 2, K \rightarrow$ pi pi decays, PoS LAT2009 (2009) 255 [arXiv:0912.2936 hep-lat]].

[23] RBC, UKQCD Collaboration, P. Boyle et al., Emerging understanding of the $\Delta I=1 / 2$ Rule from Lattice QCD, Phys.Rev.Lett. 110 (2013) 152001 [arXiv:1212.1474 [hep-1at]]. 